ARTICLE

Received 29 Sep 2013 | Accepted 13 Dec 2013 | Published 29 Jan $2014 \quad$ DOl: 10.1038/ncomms4103

\title{
Lateral $\mathrm{pH}$ gradient between OXPHOS complex IV and $\mathrm{F}_{\mathrm{O}} \mathrm{F}_{1}$ ATP-synthase in folded mitochondrial membranes
}

\author{
Bettina Rieger ${ }^{1}$, Wolfgang Junge ${ }^{2} \&$ Karin B. Busch ${ }^{1}$
}

Ion-driven ATP synthesis by rotary $F_{0} F_{1}$ ATP-synthase powers aerobic life. Since Mitchell's seminal hypothesis, this synthesis has been discussed in terms of the proton-motive force between two bulk phases, each in equilibrium. In active mitochondria, a steady proton flow cycles between pumps and the distant ATP synthase. Here we determine the lateral $\mathrm{pH}$ profile along the $\mathrm{p}$-side of cristae in situ by attaching a ratiometric fluorescent $\mathrm{pH}$-sensitive GFP variant to OXPHOS complex IV, a proton pump, and the dimeric $F_{0} F_{1}$ ATP-synthase, a proton consumer. In respiring HeLa cells, we observe that the local $\mathrm{pH}$ at $\mathrm{F}_{\mathrm{O}} \mathrm{F}_{1}$ dimers is 0.3 units less acidic than that at complex IV. This finding is consistent with the calculated $\mathrm{pH}$ profile for steady proton diffusion from CIV to $F_{0} F_{1}$. The observed lateral variation in the proton-motive force necessitates a modification to Peter Mitchell's chemiosmotic proposal. The experimental technique can be extended to other $\mathrm{pH}$-dependent reactions in membrane microcompartments.

\footnotetext{
${ }^{1}$ Division of Mitochondrial Dynamics, Fachbereich Biologie/Chemie, Universität Osnabrück, D-49076 Osnabrück, Germany. ${ }^{2}$ Niedersachsen-Professur für Biophysik, Fachbereich Biologie/Chemie, Universität Osnabrück, D-49076 Osnabrück, Germany. Correspondence and requests for materials should be addressed to K.B.B. (email: busch_k@biologie.uni-osnabrueck.de)
} 
n eukaryotic cells, adenosine triphosphate (ATP) is produced at the crista membrane of mitochondria. Cristae are flat invaginations of the mitochondrial inner membrane that extrude into the matrix space with a few tubular openings to the inter-membrane space between the inner and outer membranes ${ }^{1-3}$. The crista membrane hosts the components of the respiratory electron transport chain (complexes I-IV, alias CI-IV) and ATP synthase (complex V, $\mathrm{F}_{\mathrm{O}} \mathrm{F}_{1}$ alias $\mathrm{CV}$ ). Complexes I, III and IV pump protons from the matrix (the n-side of the membrane) into the intra-cristae space (the $\mathrm{p}$-side). The proton-motive force drives protons back via ATP synthase and ATP is formed ${ }^{4,5}$. The proton pumps and the proton-driven ATP synthase can be segregated. Complexes I-IV are mainly found in the flat sheet membrane ${ }^{6,7}$, whereas immuno-EM and EM-tomography have revealed ribbons of $\mathrm{F}_{0} \mathrm{~F}_{1}$ dimers lining the highly curved rim $^{8-12}$. These ribbons seem to be involved in folding the crista membrane. Deleting certain subunits of $\mathrm{F}_{0} \mathrm{~F}_{1}$ (termed $\mathrm{e}$ and $\mathrm{g}$ ) abolishes the dimerization, and thus the high curvature of the crista membrane without deactivating ATP synthesis ${ }^{13}$. The accumulation of ATP synthase in the highly curved rim has recently introduced a new dimension to the classical debate over localised versus delocalized (that is, chemiosmotic) proton coupling: electrostatic guidance of protons into ATP synthase. One group has postulated that the curved membrane segment concentrates protons at the concave side of the membrane, thereby generating extra proton-motive force for ATP synthesis in cristae 9 . A related process for the thylakoid membranes of chloroplasts has been coined 'Proton-Electrostatic Hypothesis for Localized Proton Coupling Bioenergetics ${ }^{14}$. Both proposals are based on the electrostatics at low ionic strength where protons and hydroxyl anions were the major charge carriers. In contrast, for a realistic situation (physiological ionic strength and steady proton flow), we expect the local $\mathrm{pH}$ at the p-side of $\mathrm{CV}$ (the proton sink) to be more alkaline and not more acidic than the $\mathrm{pH}$ at CIV (the proton source).

Here, this expectation is tested using a $\mathrm{pH}$-indicating analogue of green fluorescent protein. This analogue is fused to the p-side (intermembrane/intra-cristae space) of either CIV or dimeric CV. The local $\mathrm{pH}$ at either $\mathrm{CIV}$ or the $\mathrm{CV}$ dimer is measured in actively respiring mitochondria of HeLa cells. To intensify OXPHOS, the cells are cultured in galactose plus glutamine ${ }^{15}$ to circumvent ATP production by glycolysis. In contrast to hyperglycaemic conditions, these metabolic substrates force HeLa cells to rely on OXPHOS and CV-dimer stabilisation ${ }^{16}$. These conditions result in a lateral $\mathrm{pH}$ difference between CIV and the dimeric synthase in the intra-cristae space: the determined proton concentration at the ATP synthase molecules in the dimer ribbons around the perimeter of cristae differs from the concentration predicted by the previously described electrostatic approaches. The following treatment of diffusive proton flow under physiological conditions provides an explanation for this difference.

\section{Results}

Local pH determination at specific OXPHOS complexes. To measure local $\mathrm{pH}$ values, superecliptic pHluorin (sEcGFP) fused to subunits of OXPHOS complexes IV and V was used as a local $\mathrm{pH}$ indicator ${ }^{17}$. SEcGFP is a double emission fluorescent protein. OXPHOS subunits were tagged at their $\mathrm{C}$ terminus and checked for correct localisation in mitochondria. SEcGFP was excited with a 405-nm laser, and the fluorescence emission was recorded simultaneously at $454-474 \mathrm{~nm}$ and $508-514 \mathrm{~nm}$. The local pH was inferred from the emission ratio $\left(\mathrm{Em}_{511 / 464}\right)^{18}$. In one transiently transfected HeLa cell line, sEcGFP was fused to the C terminus of subunit $8 \mathrm{a}$ of $\mathrm{CIV}$, which is located at the p-side
(PDB ID: 2EIN). In the second cell line, sEcGFP was fused to the dimer-specific subunit e of CV, which is also located at the p-side in the rims of cristae ${ }^{19}$. The tagging of these subunits did not interfere with their localisation inside mitochondria (Fig. 1a,b), and no indication of functional impairment was found from oxygen consumption recording 20 and membrane potential measurements (Fig. 1c). The emission ratio images were intrinsically different (Fig. 1a,b). Calibration was performed
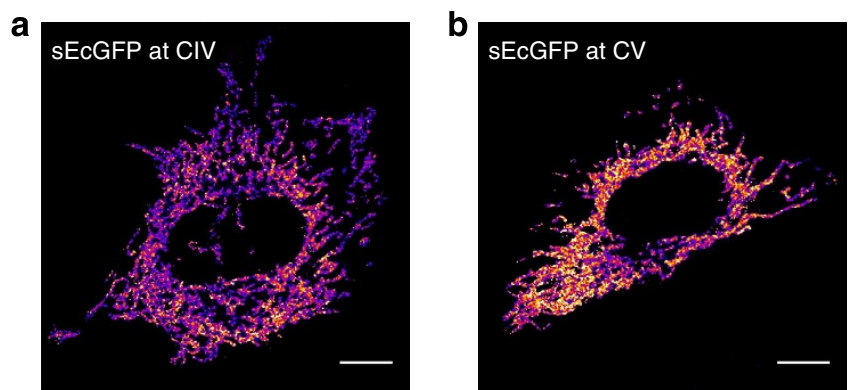

C
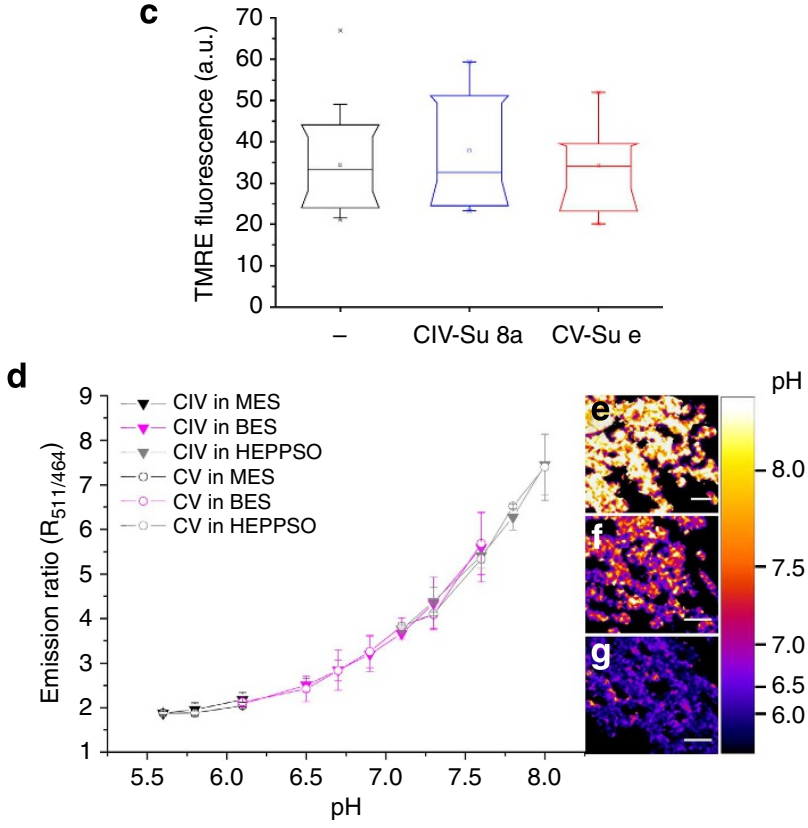

Figure 1 | Calibration of the pH indicator sEcGFP at functional subunits of OXPHOS complexes in the intra-cristae space. $(\mathbf{a}, \mathbf{b})$ Fluoro-micrographs of HeLa cells with sEcGFP at subunit 8 a of CIV (a) and subunit e of CV (b) show the mitochondrial localisation of the tagged subunits. (c) The labelling of subunits $8 \mathrm{a}(\mathrm{CIV})$ and Su e (CV) with the pH indicator sEcGFP does not influence the functionality, as indicated by the membrane potential $\Delta \Psi_{\mathrm{m}}$ measurements. The relative TMRE fluorescence in HeLa cells, CIVsEcGFP- and CV-sEcGFP-expressing cells was determined as a measure of the membrane potential $\Delta \Psi_{\mathrm{m}}$ in the presence of $5 \mathrm{nM}$ TMRE. Statistics: ( $n=2$ independent transfections with $>15$ cells each). The boxes represent the 10th to 90th percentiles with the 25th to 75th percentiles within the narrower part of the box. The vertical lines in the boxes represent the median values. The square symbols in the boxes denote the respective mean values. The error bars denote the 5 th to 95 th percentile values. The minimum and maximum values are denoted by $x$. (d) Ratiometric response of SEcGFP to the local pH. CCCP, nigericin and oligomycin were used to equilibrate the cell to the $\mathrm{pH}$ of the buffer. The fluorescence ratios at $\mathrm{pH} 6.1$, $7.1,7.3$ and $7.6 \mathrm{pH}$ were determined in two different standard buffers. Every data point represents $n=2$ independent experiments and mean values \pm s.d. from at least 10 cells. (e-g) The ratiometric $\mathrm{pH}$ values in mitochondria were colour coded according to the look-up table on the right. Scale bars: $10 \mu \mathrm{m}(\mathbf{a}, \mathbf{b}), 2 \mu \mathrm{m}(\mathbf{e}-\mathbf{g})$. 
with sEcGFP tagged to the proteins of interest in the respective cell lines under conditions in which the membranes were permeable to protons. The buffer $\mathrm{pH}$ was adjusted, and the resulting fluorescence emission ratio was recorded (Fig. 1d).

The resulting $\mathrm{pH}$ titration curves were congruent, independently of the location of attachment of the $\mathrm{pH}$ indicator and independently of the chemical nature of the buffer. For $\mathrm{pH}$ determination, a mean titration curve was used. The fluorescence emission ratio was correlated with the $\mathrm{pH}$, and the ratio images of the mitochondria were accordingly colour coded (Fig. 1e-g).

Local $\mathrm{pH}$ in respiring cristae is higher at $\mathrm{CV}$ than at CIV. We next checked the local $\mathrm{pH}$ at a cytochrome $c$ oxidase proton pump in the intra-cristae space. SEcGFP was fused to subunit $8 \mathrm{a}$ in CIV and faced the intra-cristae space. CIV is mainly found in the sheet region of cristae ${ }^{6,7}$. Concurrently, the local $\mathrm{pH}$ at $\mathrm{F}_{0} \mathrm{~F}_{1}$ ATP-synthase, which pumps protons back into the matrix under ATP synthesising conditions, was determined. SEcGFP was fused to subunit e in CV. Subunit e is involved in the dimerisation of $\mathrm{CV}^{21}$ and is also localised in the intermembrane space, mostly at the rims of cristae $^{22}$. Figure 2 illustrates the molecular localisation of the $\mathrm{pH}$ indicator (sEcGFP) fused to subunit $8 \mathrm{a}$ $(\mathrm{Su} 8 \mathrm{a})$ in $\mathrm{CIV}$ and subunit $\mathrm{e}\left(\mathrm{Su}\right.$ e) in $(\mathrm{CV})_{2}$ (Fig. 2a). Fluorescence emission ratios were recorded for sEcGFP-CIV (Su 8a) and sEcGFP-CV (Su e). The obtained fluorescence emission ratios of the location-specific sensor clearly indicate a higher proton concentration at CIV (lower $\mathrm{pH}$ ) and a lower concentration at the $\mathrm{CV}$ dimers (higher $\mathrm{pH}$ ) (Fig. 2b). These data show that the local $\mathrm{pH}$ at the proton pump (CIV) was 0.3 units more acidic than that at the proton sink $(\mathrm{CV})$. We attribute this difference to the respective proton pumping and consuming activities at the different positions of the folded cristae membrane. This difference was obtained for actively respiring mitochondria supplied with galactose.

Next, we compared different nutrition conditions. It is known that the type of energy substrate modulates the mitochondrial oxidative capacity and respiration in cancer cells, such as HeLa cells $^{15,23}$. With low glucose, the intra-cristae space was acidified, and no difference was measured between CIV and CV. Reverse ATP synthase has been suggested to maintain $\Delta \mu_{\mathrm{H}}^{+}$by pumping protons out of the matrix when respiratory proton pumping is insufficient ${ }^{24}$. The sink will then become a source. Our data are consistent with this interpretation. Supplementing cells with high glucose $\left(4.5 \mathrm{gl}^{-1}\right)$ also abrogated the difference in the lateral $\mathrm{pH}$ between the source and sink, but the $\mathrm{pH}$ generally increased (Fig. 2c). In these conditions, cells gain ATP mostly by substrate chain phosphorylation in glycolysis, and both respiration ${ }^{16,23}$ and proton pumping are reduced. In addition, hyperglycaemia obviously is accompanied by a decrease in the number of $\mathrm{F}_{0} \mathrm{~F}_{1}$ ATP-synthase dimers ${ }^{16}$.

Inhibitors and protonophores eliminate the lateral $\mathrm{pH}$ gradient. We also tested the significance of the lateral $\mathrm{pH}$ difference in respiring cells by using inhibitors and protonophores. The difference was expected to disappear when the membrane was permeable to protons (by the addition of the protonophore CCCP in this study) or when the proton cycling was blocked at $\mathrm{F}_{0} \mathrm{~F}_{1}$ ATP-synthase (by the addition of oligomycin in this study). The cell structure was not obviously altered upon the addition of either CCCP or oligomycin (Fig. 3a,b) within the first $50 \mathrm{~min}$. Both agents equilibrated the local $\mathrm{pH}$ values at $\mathrm{CIV}$ and $\mathrm{CV}$, as projected. The $\mathrm{pH}$ values shifted into acidity (by oligomycin, especially after 30-50 min) and towards a medium $\mathrm{pH}$ (by CCCP), also as expected (Fig. 3c). Total equilibration with the medium $\mathrm{pH}$ was only observed after prolonged CCCP treatment, a
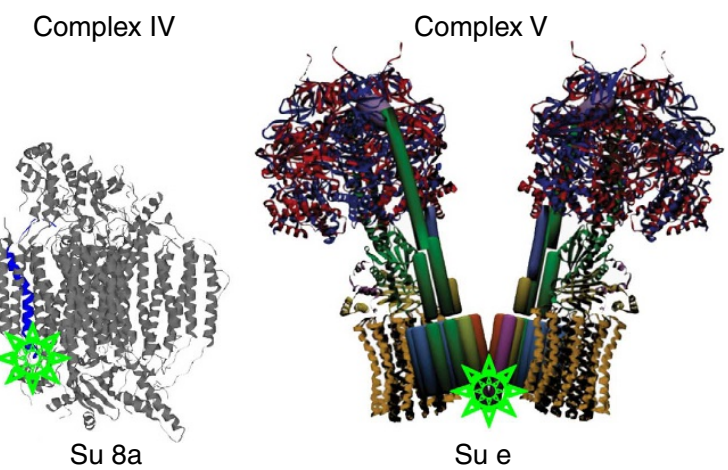

b

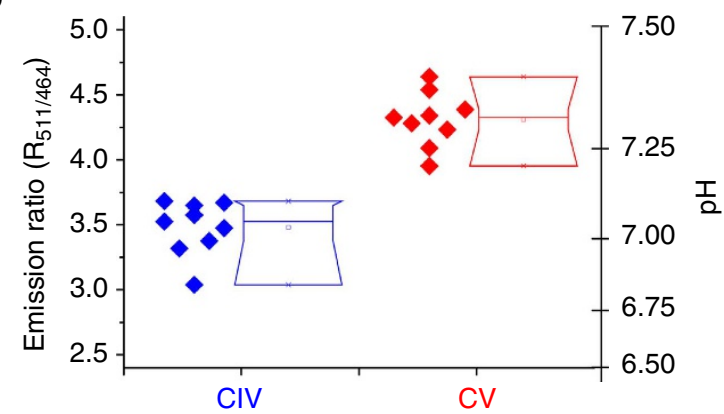

C

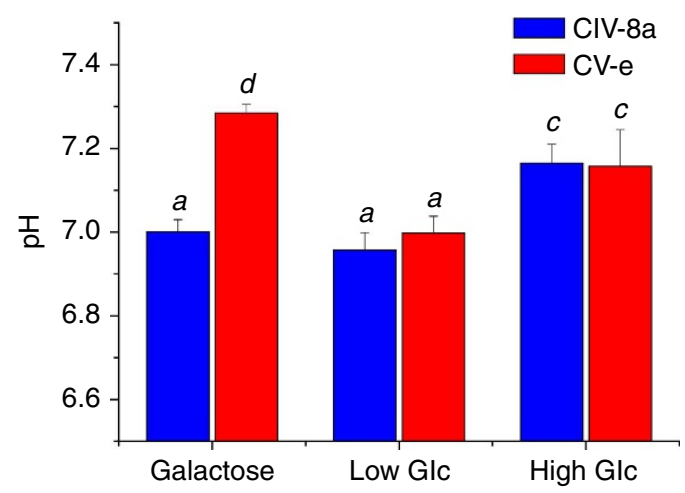

Figure 2 | Local pH values at CIV and $F_{0} F_{1}$ ATP-synthase in actively respiring HeLa mitochondria. (a) Structural models of complex IV and the CV dimer attached to sEcGFP (green). (b) Box-and-whisker plot and mean data points (each data point represents the mean of 5 cells in one experiment) showing the fluorescence emission ratio (left scale) and the corresponding local pH (right scale) at the proton pump (CIV) and at $F_{0} F_{1}$ synthase (CV) in the intra-cristae space of transiently transfected cells. The boxes represent the 10th to 90th percentiles with the 25th to 75th percentiles within the narrower part of the box. The vertical lines in the boxes represent the median values. The square symbols in the boxes denote the respective mean values. The error bars denote the 1st and 99th percentile values. The minimum and maximum values are denoted by $x$.

The local $\mathrm{pH}$ was inferred from the emission ratio using the mean titration curve from Fig. 1. (c) Mean $\mathrm{pH}$ values at $\mathrm{CIV}$ and $\mathrm{CV}$ under various nutrition conditions (mean \pm s.e.m.; significance levels determined by a one-way ANOVA $(a, b): P \leq 0.05 ;(a-c): P \leq 0.01 ;(a-d): P \leq 0.001 ; n=9$ independent experiments with 45 determined values for respiration and low glucose; $n=2$ independent measurements and 14 data points for high glucose). Media: respiration with glutamine $(2 \mathrm{mM})$ and galactose $(10 \mathrm{mM})$; glycolysis with a low $(5.5 \mathrm{mM})$ and high glucose $(25 \mathrm{mM})$ supply.

which led to mitochondrial fragmentation in some cells and changes in cell shape. The induced acidification of the intermembrane space after prolonged oligomycin treatment is 
expected because $\mathrm{F}_{0} \mathrm{~F}_{1}$ ATP-synthase (the sink) is blocked by oligomycin while the proton pumps (source) of the galactosegrown cells still function. These $\mathrm{pH}$ changes will continue until a feedback mechanism stops them. In comparison with the CCCP treatment, the inhibition of CIV by KCN caused an even stronger $\mathrm{pH}$ increase in the intra-cristae space, as expected if the proton pumping activity of this terminal oxidase was effectively blocked. $\mathrm{KCN}$ led to rapid mitochondrial fragmentation and cell shape changes (Fig. 3d), which are unwanted side effects. The $\mathrm{pH}$ results provide further evidence in favour of the localisation of the $\mathrm{pH}$ sensor at the p-side of the membrane.
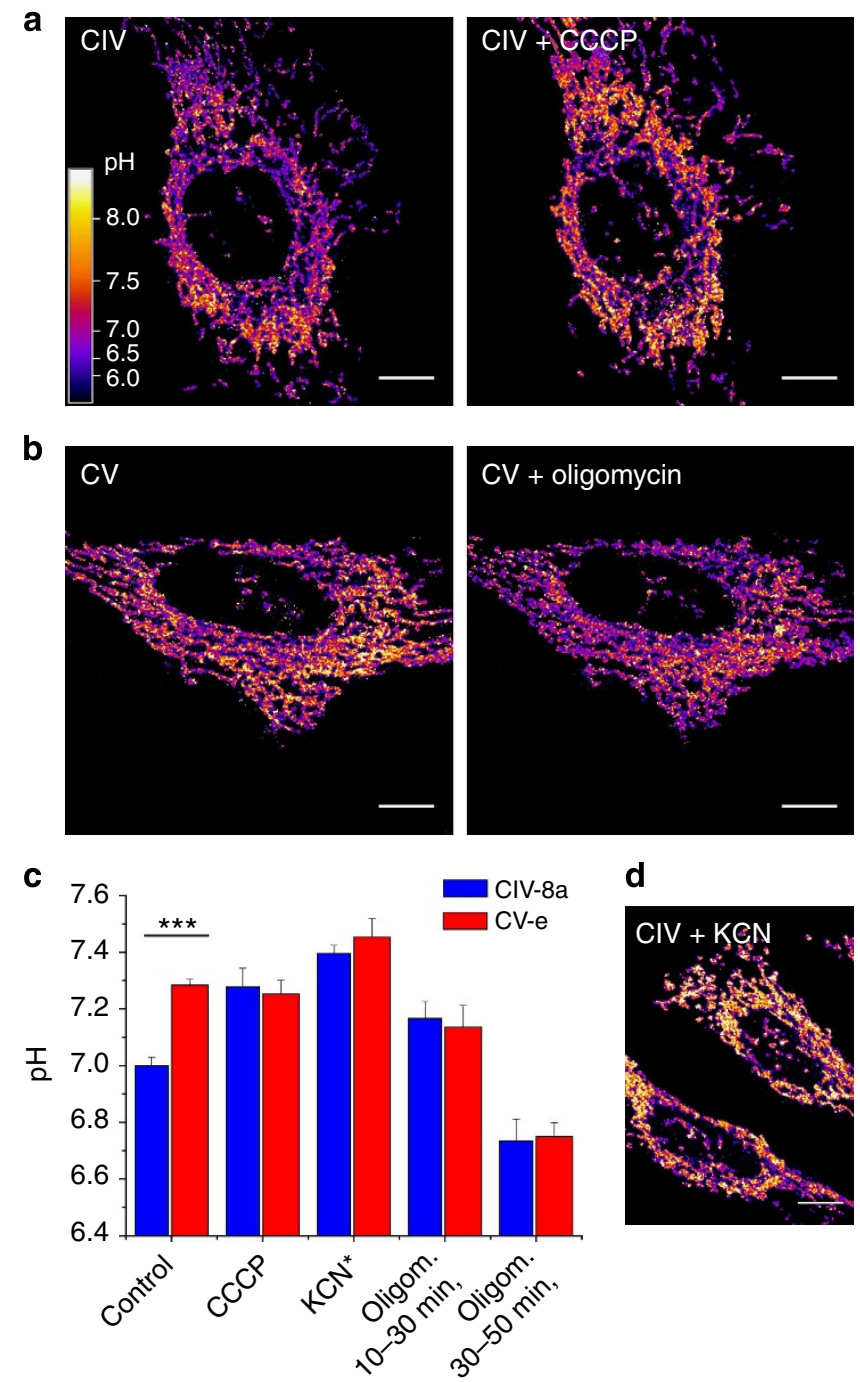

Figure 3 | Protonophore and inhibitor effects on the local pH values in the intra-cristae space. Effects of short circuiting ((a), $10 \mu \mathrm{M} \mathrm{CCCP)} \mathrm{and}$ blocking ((b), $5 \mu \mathrm{g} \mathrm{ml}^{-1}$ oligomycin) of the cyclic flow of protons between CIV and CV. (c) Ratiometrically determined local pH under the above mentioned conditions compared with that in untreated cells (control). The $\mathrm{pH}$ of the medium was 7.5. CCCP $(10 \mu \mathrm{M}): n=4$ independent experiments and 20 cells; $\mathrm{KCN}(3 \mathrm{mM}): n=1$ experiment and 7 cells; 10-30 min oligomycin: $\mathrm{n}=4$ independent experiments and 20 cells; 30-50 min oligomycin: $n=4$ independent experiments and 20 cells. *Because of the rapid fragmentation of $\mathrm{KCN}$-treated mitochondria, these measurements were not pursued. Mean \pm s.e.m.; significance levels were determined between the CIV values and the $C V$ values for each treatment using a one-way ANOVA ${ }^{\star \star \star} P \leq 0.001$ (d) Fluoro-micrograph showing fragmented mitochondria after $\mathrm{KCN}$ treatment ( $3 \mathrm{mM}, 10 \mathrm{~min}$ ) in CIVSu8a-sEcGFP-transfected cells. Scale bars, $10 \mu \mathrm{m}$.
Diffusion limitation counteracts the lateral $\mathrm{pH}$ equilibration. When the proton pumping and consuming activities of the OXPHOS complexes are located at different positions in the flat crista membrane, the diffusion of protons becomes an important issue. The stationary proton diffusion between sources and sinks in a disk-shaped crista was simulated using the following simplifying assumptions, which are illustrated in Fig. 4:

1. The intra-cristae space is a flat disk of radius $R$ and thickness $h$.

2. Proton pumps are homogeneously distributed over the flat portions of the disk membrane.

3. Dimers of CV line the perimeter of the disk.

4. Cristae junctions are obstructed.

5. The total flux of protons into the ring of ATP synthase molecules, $I_{\text {tot }}(R)\left(\mathrm{mol} \times \mathrm{s}^{-1}\right)$, is constant due to the dominant role of the electric driving force.

6. Lateral proton flow between CIV and CV is diffusive without drift, due to the high ionic strength.

The total flux at radius $r, I_{\text {tot }}(r)\left(\mathrm{mol} \mathrm{s}^{-1}\right)$, depends on the diffusive flux density at this radius, $J(r)\left(\mathrm{mol} \times \mathrm{m}^{-2} \times \mathrm{s}^{-1}\right)$, as follows:

$$
I_{\text {tot }}(r)=2 \pi r \cdot h \cdot J(r) \text { and } I_{\text {tot }}(R)=2 \pi R \cdot h \cdot J(R)
$$

The total fluxes at the given radii $r$ and $R$ are proportional to the respective disk areas that are homogeneously filled with proton pumps such that

$$
\frac{I_{\mathrm{tot}}(r)}{I_{\mathrm{tot}}(R)}=\frac{r^{2}}{R^{2}}=\frac{2 \pi r \cdot J(r)}{2 \pi R \cdot J(R)}
$$

and hence

$$
J(r)=\frac{r}{R} \cdot J(R)
$$

The diffusive flux density across any ring of radius $r$, $J(r)\left(\mathrm{mol} \times \mathrm{m}^{-2} \times \mathrm{s}^{-1}\right)$, obeys Fick's second law:

$$
J(r)=-D \cdot \frac{\partial\left[H^{+}(r)\right]}{\partial r}
$$

where $D\left(\mathrm{~m}^{2} \times \mathrm{s}^{-1}\right)$ is the diffusion coefficient of the protons.

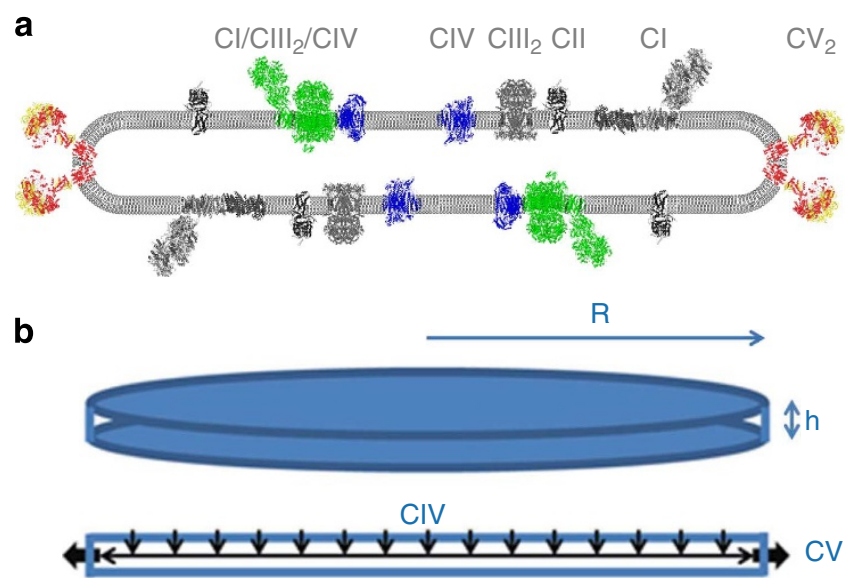

Figure 4 | Schematic representation of a crista with proton flow.

(a) Cross section with proton pumps (CIV in blue as a monomer and in the supercomplex $\mathrm{Cl}-\mathrm{CIII}{ }_{2}-\mathrm{CIV}$ ) in the flat membrane and proton sinks (CV dimers, red) at the curved rim. (b) Overview and cross section with black arrows illustrating the proton flow (see text). 
With equation (2), the solution of Fick's equation becomes the following:

$$
\left[H^{+}(r)\right]=\left[H^{+}(0)\right]-\frac{r^{2}}{R^{2}} \cdot \frac{I_{\mathrm{tot}}(R)}{4 \pi \cdot h \cdot D}
$$

Averaging over a homogenous distribution of CIV over the disk leads to the following solution:

$$
\left\langle\left[H^{+}(r)\right]\right\rangle=\left[H^{+}(0)\right]-\frac{1}{2} \cdot \frac{I_{\mathrm{tot}}(R)}{4 \pi \cdot h \cdot D}
$$

With the given assumptions, the calculated proton concentration drops parabolically from the centre of the disk-shaped membrane towards the ring of $\mathrm{CV}$ dimers at the perimeter. Using equation (5), we calculated the drop in the proton concentration from the centre to the perimeter of a crista $(R=0.5 \mu \mathrm{m})$ using a reasonable assumption for the total flux $\left(I_{\mathrm{tot}}(R)=50 \cdot 10^{-21} \mathrm{~mol} \times \mathrm{s}^{-1}\right)$, the thickness of the intra-crista space $(h=10 \mathrm{~nm})$, and the diffusion coefficient of a proton $\left(D \approx 5 \cdot 10^{-9} \mathrm{~m}^{2} \times \mathrm{s}^{-1}\right)$ in the crowded and diffusion-obstructed intra-crista space (see ref. 25).

The calculated concentration drop was $80 \mathrm{nM}$ from the centre of the crista disk $(\mathrm{pH}$ 6.8) to the perimeter. This drop is equivalent to a $\mathrm{pH}$ increase of 0.7 units from the centre (assumed $\mathrm{pH}$ 6.8) to the rim (calculated $\mathrm{pH}$ 7.5). The mean concentration drop was $40 \mathrm{nM}$ (see equation (6)). The calculated values follow the same pattern as in the experiments; they are not meant to match quantitatively because the idealizations underlying the above solution do not strictly hold. ATP synthase molecules are not restricted to the perimeter at the rims of cristae ${ }^{26}$, the proton pumps may be non-homogeneously distributed over the flat surfaces, and the crista junction may not be perfectly proton tight. All these complications, however, do not invalidate the finding that the proton concentration at the inlet of the ATP synthase dimers is lower but not higher than the concentration elsewhere in the intra-cristae space.

\section{Discussion}

In the present study, we experimentally determined the lateral $\mathrm{pH}$ difference between the respiratory chain complex cytochrome $c$ oxidase (proton pump) and $\mathrm{F}_{0} \mathrm{~F}_{1}$ ATP-synthase dimers (proton consumer). The two OXPHOS complexes are located at different positions in flat, hollow crista discs. CIV is mainly found in the sheet region ${ }^{6,7}$, while $\mathrm{F}_{0} \mathrm{~F}_{1}$ ATP-synthase dimers shape the rim $8,27-29$. Under respiratory conditions, the actual $\mathrm{pH}$ at the $\mathrm{F}_{0} \mathrm{~F}_{1}$ dimers at the rims of cristae was higher than the $\mathrm{pH}$ at the proton pump in the sheet region. Thus, the specific architecture of cristae with dimerisation and ribbon formation by ATP synthase does not enhance but rather diminishes the local proton-motive force for ATP synthesis. This result qualifies the contradictory claims of two other groups ${ }^{9,14}$. In his chemiosmotic hypothesis ${ }^{4,5}$, Peter Mitchell considered two bulk phases, and he assumed a constant proton-motive force across the coupling membrane. According to the data and the diffusion calculated in the present work, this assumption does not apply for a steady proton flow between a proton pump and a remote dimeric ATP synthase. For mitochondria, however, the amendment to Mitchell's original concept is minor. While the entropic contribution to the proton-motive force $(-2.3 \mathrm{RT} \Delta \mathrm{pH})$ varies laterally, the electric component $(\mathrm{F} \Delta \Psi)$ is not affected due to the high ionic strength. Slightly lowering the transmembrane $\mathrm{pH}$ difference at the ATP synthase dimers on the rim of cristae is intrinsically unfavourable to ATP production by CV dimers. By folding the cristae, however, dimerisation increases the packing density of the energy-transducing membrane and therewith the ATP production per volume. Some CV molecules reside in cristae sheets $^{7,26}$. In hyperglycaemic conditions, which have been reported to decrease the number of $\mathrm{CV}$ dimers, the $\mathrm{pH}$ difference between CIV and CV disappears, as shown here. In addition, inhibitor effects eliminated the lateral difference between CIV and CV, as expected. Effective inhibition of the respiratory chain electron flow due to CIV inhibition by KCN also increased the intra-cristae space $\mathrm{pH}$ but was accompanied by unwanted ultrastructural changes and mitochondrial fragmentation. In contrast, during oligomycin treatment, acidification of the IMS proceeded due to the continuing activity of the proton pump. At the same time, the lateral $\mathrm{pH}$ gradient between CIV and CV vanished because of the inhibition of the sink, which switches the system from a steady state to equilibrium. In conclusion, the intra-cristae space obviously is a microcompartment with different structural and functional domains.

Recently, $10-80 \mathrm{~s} \mathrm{pH}$ pulses were found in the mitochondrial matrix ${ }^{30,31}$. The pulses should correspond to an alkalinisation of the intermembrane space. We cannot rule out the possibility that such pulses were recorded without being recognized. It is certainly challenging to address this possibility in the future.

The observations in the present study ought to be of interest for ATP synthesis in all kingdoms of life. Chloroplasts have achieved even higher packing densities in their thylakoid membranes than found in cristae membranes. Despite its intricate folding, the thylakoid membrane forms a simply connected sheet in which the electric potential difference is delocalised over more than 10,000 ATP synthase molecules (see ref. 32); however, the $\mathrm{pH}$ difference may also vary laterally, as established for mitochondria in this work. In alkaliphilic bacteria, the close proximity of complex $\mathrm{V}$ to a proton pump may be essential for ATP synthesis to occur with virtually zero bulk-to-bulk protonmotive force (see refs $12,27,33-36$ ).

\section{Methods}

Plasmid constructs. For eukaryotic expression, the full-length protein-coding region of superecliptic pHluorin (F64L/S65T/S147D/N149Q/V163A/S175G/S202F/ Q204T/A206T) (a gift from Prof. Jürgen Klingauf) was inserted by PCR amplification into a modified pSEMS-26 $\mathrm{m}$ vector from NEB Biosciences (formerly Covalys Biosciences) using the following primers: forward $5^{\prime}$-GCGCGAATTCACCATGAG TAAAGGAGAAGAACTTTTCACTGGAG-3' and reverse 5-GCGCGGATCCACCGGTTTTGTATAGTTCATCCATGCCA TG-3', with an $E c o$ RI (forward) and a Bam $\mathrm{HI}$ (reverse) restriction site (underlined). The respective subunits of OXPHOS complexes were fused with their $\mathrm{C}$ termini to the $\mathrm{N}$ terminus of sEcGFP using the following primers: $5^{\prime}$-GCGCGATATCATGTTGGCTACCAGGG-3' (forward) and 5'-GCGCGAATTCCTTCTTCCACTCGTTCTTTTC G-3' (reverse) for CIV-Su 8a, and $5^{\prime}$-GCGTAAGATATCATGGTGCCACCGGTGCAGG-3' (forward) and $5^{\prime}$-GCAATTGAATTCCTTTAATATGCTGTCATCTTCTG- $3^{\prime}$ (reverse) for CV Su e (the EcoRV and EcoRI restriction sites are underlined $)^{20}$. PCR primers were purchased from Sigma Aldrich. Enzymes, restriction buffer and BSA were purchased from NEB Biosciences.

Cell culture and treatments. HeLa cells, purchased from DSMZ (Deutsche Sammlung von Mikroorganismen und Zellkulturen $\mathrm{GmbH}$ ), were cultured in minimal essential medium with Earle's salts (MEM, PAA Lab GmbH, E15-888) with $5.5 \mathrm{mM}$ glucose, $2 \mathrm{mM}$ stable glutamine and sodium bicarbonate, supplemented with $10 \%(\mathrm{v} / \mathrm{v})$ fetal bovine serum (FBS) superior (Biochrom AG), $1 \%$ MEM nonessential amino acids (NEA, Biochrom AG) and 1\% 4-(2-hydroxyethyl)piperazine-1-ethanesulfonic acid (HEPES, PAA Lab GmbH) at $37^{\circ} \mathrm{C}$ with $5 \% \mathrm{CO}_{2}$. The cells were split 2-3 times a week using trypsin/EDTA (Biochrom), supplemented with HEPES (PAA), sodium bicarbonate (PAA), penicillin/streptomycin (Biochrom) and PBS (Biochrom). The cells were transfected at $60-80 \%$ confluence with $5 \mu \mathrm{g}$ DNA in $2.5 \mathrm{ml}$ fresh medium. For the analysis of actively respiring mitochondria, the glucose medium was replaced with galactose medium (Dulbecco's MEM = DMEM (PAA, E15-079)) with NEA, without glucose, and supplemented with $10 \mathrm{mM}$ galactose (Sigma), $4 \mathrm{mM}$ glutamine (Biochrom), $10 \%$ (v/v) fetal bovine serum (FBS) superior (Biochrom), sodium bicarbonate (PAA) and HEPES (PAA) for at least 2 days. For the analysis of hyperglycaemic mitochondria, the glucose medium was replaced by DMEM (PAA, E15-079), supplemented with $25 \mathrm{mM}$ glucose (Sigma), $2 \mathrm{mM}$ glutamine, $10 \%(\mathrm{v} / \mathrm{v})$ fetal bovine serum (FBS) superior, sodium bicarbonate (PAA) and HEPES (PAA) for at least 2 days. For the protonophore and inhibitor studies, the cells were additionally treated with $10 \mu \mathrm{M}$ CCCP (carbonyl cyanide m-chlorophenyl hydrazone, Enzo Life) for at least $30 \mathrm{~min}$ and $5 \mu \mathrm{g} \mathrm{ml}^{-1}$ oligomycin (Enzo Life, times indicated) or $3 \mathrm{mM} \mathrm{KCN}$ (potassium cyanide, Sigma) for 10-30 min. 
Ratio imaging microscopy for $\mathbf{p H}$ determination. Two days after the transfection with $\mathrm{sEcGFP}$ fusion constructs, the $\mathrm{pH}$ of the transfected cells was recorded in situ by confocal fluorescence emission ratio imaging (inverted Olympus Fluoview FV1000). The cLSM was equipped with a $\times 60$ oil immersion objective (UPLSAPO oil, NA 1.35, $\infty / 0.17 /$ FN26.5) and two spectral detectors. All measurements were performed at room temperature in fresh growth medium. Ratio images were obtained simultaneously using the NIR laser (ex. $405 \mathrm{~nm}$ ) of the microscope (em. $454-474 \mathrm{~nm}$ and $508-514 \mathrm{~nm}$ ).

In situ $\mathbf{~ p H}$ calibration. For $\mathrm{pH}$ calibration, cells were perfused initially for 3-5 min with PBS (PAA, pH 7.0-7.5 with $\mathrm{CaCl}_{2}$ and $\mathrm{MgCl}_{2}$ ). Then, $\mathrm{PBS}$ was exchanged for MES (pH 5.6-6.1), BES (pH 6.1-7.6) or HEPPSO (pH 7.1-8.0) buffer, which was adjusted to the desired $\mathrm{pH}$ by $1 \mathrm{M} \mathrm{HCl}$ or $\mathrm{NaOH}$, and supplemented with the following compounds: $125 \mathrm{mM} \mathrm{KCl}, 20 \mathrm{mM} \mathrm{NaCl}, 0.5 \mathrm{mM} \mathrm{CaCl}_{2}, 0.5 \mathrm{mM} \mathrm{MgSO}_{4}$, $20 \mu \mathrm{M}$ CCCP, $1 \mu \mathrm{M}$ nigericin and $5 \mu \mathrm{g} \mathrm{ml}^{-1}$ oligomycin. Images were obtained within 15-45 min of incubation. Nigericin was purchased from Enzo Life. MES was purchased from Biomol, and BES and HEPPSO were purchased from Sigma. Other commonly used chemicals were purchased from Roth.

Membrane potential measurements. The membrane potential was determined with $5 \mathrm{nM}$ TMRE and without quenching after $30 \mathrm{~min}$ of incubation with verapamil $(10 \mu \mathrm{M})$ to block the multidrug transporter. In brief, verapamil and $5 \mathrm{nM}$ TMRE were added to the incubation medium for $30 \mathrm{~min}$ at $37^{\circ} \mathrm{C}$. Then, the cells were mounted into a chamber with fresh incubation medium containing $5 \mathrm{nM}$ TMRE and $10 \mu \mathrm{M}$ verapamil for microscopy studies. Thus, measurements were performed using equilibrated dye. The fluorescence of mitochondrial TMRE in the mitochondrial regions of interest was then determined using the same settings (gain and fluorescence excitation power) in all samples. Because the cells were transiently transfected, an internal control was always available. Data were analysed by using ImageJ software (NIH Image, http://rsb.info.nih.gov/nih image/ index.html). The mean grey values of mitochondrial TMRE (lines along site mitochondria $\sim 3 \mu \mathrm{m}$, pixel size: $1 \mathrm{px}=0.166 \mu \mathrm{m}$ site) were determined. Because the uncoupling of mitochondria after the addition of CCCP $(10 \mu \mathrm{M})$ led to an almost complete disappearance of the TMRE signal, we concluded that the measurements were performed without quenching.

Data analysis. The data were analysed by using ImageJ software (NIH Image, $\mathrm{http} / / / \mathrm{rsb}$.info.nih.gov/nih image/index.html) according to the procedure from Morgan et al. ${ }^{37}$ In brief, to determine intracellular $\mathrm{pH}$ maps, backgroundsubtracted images were processed using the sigma filter option of the ImageJ software (pixel radius $=1$, pixels used within 2 sigmas, minimum pixel fraction $=0.4$ ) and scaled up to 5 . Then, a threshold level for the converted 32-bit images was determined interactively for each cell. The threshold was determined only for the images recorded at $\lambda_{\mathrm{e}}=508-514 \mathrm{~nm}$ because these images had higher signal/noise ratios and were divided by the images with the lower signal/noise ratio. The background was set to NaN. For the final image, another threshold was set to $>1$, and the ratio was determined. All the background pixels were set to NaN in the images; within the cells, each pixel represents the ratio of the emission at $508-514 \mathrm{~nm}$ to that at $454-474 \mathrm{~nm}$. The Boltzmann fit was chosen here for fitting the sigmoidal curves of the calibration data, in accordance with Grynkiewicz ${ }^{38}$, who described the relation between analyte concentration and fluorescence and fluorescence ratios. The fitting was performed using Origin 6.0 (OriginLab Corp., Northhampton, MA, USA).

\section{References}

1. Mannella, C. A. et al. Topology of the mitochondrial inner membrane: dynamics and bioenergetic implications. IUBMB Life 52, 93-100 (2001).

2. You, C. et al. Self-controlled monofunctionalization of quantum dots for multiplexed protein tracking in live cells. Angew Chem. 49, 4108-4112 (2010).

3. Sun, M. G. et al. Correlated three-dimensional light and electron microscopy reveals transformation of mitochondria during apoptosis. Nat. Cell Biol. 9, 1057-1065 (2007).

4. Mitchell, P. Coupling of photophosphorylation to electron and hydrogen transfer by a chemiosmotic type of mechanism. Nature 191, 144-148 (1961).

5. Mitchell, P. Chemiosmotic coupling in oxidative and photosynthetic phosphorylation. Physiol.Rev. 41, 445-502 (1966).

6. Vogel, F., Bornhovd, C., Neupert, W. \& Reichert, A. S. Dynamic subcompartmentalization of the mitochondrial inner membrane. J. Cell Biol. 175, 237-247 (2006).

7. Wilkens, V., Kohl, W. \& Busch, K. Restricted diffusion of OXPHOS complexes in dynamic mitochondria delays their exchange between cristae and engenders a transitory mosaic distribution. J. Cell Sci. 126, 103-116 (2013).

8. Dudkina, N. V., Heinemeyer, J., Keegstra, W., Boekema, E. J. \& Braun, H. P. Structure of dimeric ATP synthase from mitochondria: an angular association of monomers induces the strong curvature of the inner membrane. FEBS Lett. 579, 5769-5772 (2005).
9. Strauss, M., Hofhaus, G., Schroder, R. R. \& Kuhlbrandt, W. Dimer ribbons of ATP synthase shape the inner mitochondrial membrane. EMBO J. 27, 1154-1160 (2008).

10. Davies, K. M. et al. Macromolecular organization of ATP synthase and complex I in whole mitochondria. Proc. Natl Acad. Sci. USA 108, 14121-14126 (2011).

11. Rabl, R. et al. Formation of cristae and crista junctions in mitochondria depends on antagonism between Fcj1 and Su e/g. J. Cell Biol. 185, 1047-1063 (2009).

12. Allen, R. D., Schroeder, C. C. \& Fok, A. K. An investigation of mitochondrial inner membranes by rapid-freeze deep-etch techniques. J. Cell Biol. 108, 2233-2240 (1989).

13. Paumard, P. et al. The ATP synthase is involved in generating mitochondrial cristae morphology. EMBO J. 21, 221-230 (2002).

14. Lee, J. W. Proton-electrostatic hypothesis for localized proton coupling bioenergetics. Bioenergetics 1, 104 (2012).

15. Reitzer, L. J., Wice, B. M. \& Kennell, D. Evidence that glutamine, not sugar, is the major energy source for cultured HeLa cells. J. Biol. Chem. 254, 2669-2676 (1979).

16. Domenis, R., Bisetto, E., Rossi, D., Comelli, M. \& Mavelli, I. Glucose-modulated mitochondria adaptation in tumor cells: a focus on ATP synthase and inhibitor factor 1. Int. J. Mol. Sci. 13, 1933-1950 (2012).

17. Miesenbock, G., De Angelis, D. A. \& Rothman, J. E. Visualizing secretion and synaptic transmission with $\mathrm{pH}$-sensitive green fluorescent proteins. Nature 394, 192-195 (1998).

18. Narendra, D. P. \& Youle, R. J. Targeting mitochondrial dysfunction: role for PINK1 and Parkin in mitochondrial quality control. Antioxid. Redox. Signal 14, 1929-1938 (2011).

19. Belogrudov, G. I., Tomich, J. M. \& Hatefi, Y. Membrane topography and nearneighbor relationships of the mitochondrial ATP synthase subunits e, f, and g. J. Biol. Chem. 271, 20340-20345 (1996).

20. Muster, B. et al. Respiratory chain complexes in dynamic mitochondria display a patchy distribution in life cells. PLoS One 5, el1910 (2010).

21. Arnold, I., Pfeiffer, K., Neupert, W., Stuart, R. A. \& Schagger, H. Yeast mitochondrial F1F0-ATP synthase exists as a dimer: identification of three dimer-specific subunits. EMBO J. 17, 7170-7178 (1998).

22. Davies, K. M. et al. Macromolecular organization of ATP synthase and complex I in whole mitochondria. Proc. Natl Acad. Sci. USA. 108, 14121-14126 (2011).

23. Rossignol, R. et al. Energy substrate modulates mitochondrial structure and oxidative capacity in cancer cells. Cancer Res. 64, 985-993 (2004).

24. Campanella, M., Parker, N., Tan, C. H., Hall, A. M. \& Duchen, M. R. IF(1): setting the pace of the $\mathrm{F}(1) \mathrm{F}(\mathrm{o})$-ATP synthase. Trends Biochem. Sci. 34, 343-350 (2009).

25. Zhang, C. et al. Water at hydrophobic interfaces delays proton surface-to-bulk transfer and provides a pathway for lateral proton diffusion. Proc. Natl Acad. Sci. USA 109, 9744-9749 (2012).

26. Rabl, R. et al. Formation of cristae and crista junctions in mitochondria depends on antagonism between Fcj1 and Su e/g. J. Cell Biol. 185, 1047-1063 (2009).

27. Paumard, P. et al. The ATP synthase is involved in generating mitochondrial cristae morphology. EMBO J. 21, 221-230 (2002).

28. Giraud, M. F. et al. Is there a relationship between the supramolecular organization of the mitochondrial ATP synthase and the formation of cristae? Biochim. Biophys. Acta 1555, 174-180 [pii] (2002).

29. Minauro-Sanmiguel, F., Wilkens, S. \& Garcia, J. J. Structure of dimeric mitochondrial ATP synthase: novel F0 bridging features and the structural basis of mitochondrial cristae biogenesis. Proc. Natl Acad. Sci. USA 102, 12356-12358 (2005).

30. Azarias, G. \& Chatton, J. Y. Selective ion changes during spontaneous mitochondrial transients in intact astrocytes. PLoS One 6, e28505 (2011).

31. Santo-Domingo, J., Giacomello, M., Poburko, D., Scorrano, L. \& Demaurex, N. OPA1 promotes $\mathrm{pH}$ flashes that spread between contiguous mitochondria without matrix protein exchange. $E M B O ~ J .32,1927-1940$ (2013).

32. Schönknecht, G., Althoff, G. \& Junge, W. The electric unit size of thylakoid membranes. FEBS Lett. 277, 65-68 (1990).

33. Mulkidjanian, A. Y., Heberle, J. \& Cherepanov, D. A. Protons @ interfaces: implications for biological energy conversion. Biochim. Biophys. Acta 1757, 913-930 (2006).

34. Cherepanov, D. A., Junge, W. \& Mulkidjanian, A. Y. Proton transfer dynamics at the membrane/water interface: dependence on the fixed and mobile $\mathrm{pH}$ buffers, on the size and form of membrane particles, and on the interfacial potential barrier. Biophys. J. 86, 665-680 (2004).

35. Mitchell, P. Coupling of phosphorylation to electron and hydrogen transfer by a chemi-osmotic type of mechanism. Nature 191, 144-148 (1961).

36. Mitchell, P. Chemiosmotic coupling in oxidative and photosynthetic phosphorylation. Biol. Rev. Camb. Philos. Soc. 41, 445-502 (1966). 
37. Morgan, B., Sobotta, M. C. \& Dick, T. P. Measuring E(GSH) and H2O2 with roGFP2-based redox probes. Free. Radic. Biol. Med. 51, 1943-1951 (2011).

38. Grynkiewicz, G., Poenie, M. \& Tsien, R. Y. A new generation of Ca2 + indicators with greatly improved fluorescence properties. J. Biol. Chem. 260, 3440-3450 (1985).

\section{Acknowledgements}

We thank Jacob Piehler for continuous support and Wladislaw Kohl for excellent technical assistance. This work was supported by a DFG grant to Karin Busch (Bu2288/1-1) and by the SFB 944. Wolfgang Junge was endowed with a post-retirement grant (Niedersachsenprofessur) by the Government of Lower Saxony.

\section{Author contributions}

B.R. designed experiments and analysed data. W.J. and K.B.B. designed experiments, analysed data and wrote the manuscript. W.J. performed calculations. K.B.B. developed strategies.

\section{Additional information}

Competing financial interests: The authors declare no competing financial interests.

Reprints and permission information is available online at http://npg.nature.com/ reprintsandpermissions/

How to cite this article: Rieger, B. et al. Lateral pH gradient between OXPHOS complex IV and $\mathrm{F}_{0} \mathrm{~F}_{1}$ ATP-synthase in folded mitochondrial membranes. Nat. Commun. 5:3103 doi: $10.1038 /$ ncomms4103 (2014). 